Article

\title{
DNA Methylation Contributes to the Differential Expression Levels of Mecp 2 in Male Mice Neurons and Astrocytes
}

\author{
Vichithra R.B. Liyanage ${ }^{1,2}$, Carl O. Olson ${ }^{1,2}$, Robby M. Zachariah ${ }^{1,2}$, James R. Davie ${ }^{2}$ and \\ Mojgan Rastegar $1,2, * \mathbb{C}$ \\ 1 Regenerative Medicine Program, Max Rady College of Medicine, Rady Faculty of Health Sciences, \\ University of Manitoba, Winnipeg, MB R3E 0J9, Canada; vichy.mb@gmail.com (V.R.B.L.); \\ carl.olson@umanitoba.ca (C.O.O.); robbymz@gmail.com (R.M.Z.) \\ 2 Department of Biochemistry and Medical Genetics, Max Rady College of Medicine, Rady Faculty of Health \\ Sciences, University of Manitoba, Winnipeg, MB R3E 0J9, Canada; jim.davie@umanitoba.ca \\ * Correspondence: mojgan.rastegar@umanitoba.ca; Tel.: +1-(204)-272-3108; Fax: +1-(204)-789-3900
}

Received: 21 February 2019; Accepted: 11 April 2019; Published: 14 April 2019

check for updates

\begin{abstract}
Methyl CpG binding protein-2 (MeCP2) isoforms (E1 and E2) are important epigenetic regulators in brain cells. Accordingly, MeCP2 loss- or gain-of-function mutation causes neurodevelopmental disorders, including Rett syndrome (RTT), MECP2 duplication syndrome (MDS), and autism spectrum disorders (ASD). Within different types of brain cells, highest MeCP2 levels are detected in neurons and the lowest in astrocytes. However, our current knowledge of Mecp2/MeCP2 regulatory mechanisms remains largely elusive. It appears that there is a sex-dependent effect in X-linked MeCP2-associated disorders, as RTT primarily affects females, whereas MDS is found almost exclusively in males. This suggests that Mecp 2 expression levels in brain cells might be sex-dependent. Here, we investigated the sex- and cell type-specific expression of Mecp 2 isoforms in male and female primary neurons and astrocytes isolated from the murine forebrain. Previously, we reported that DNA methylation of six Mecp2 regulatory elements correlated with Mecp2 levels in the brain. We now show that in male brain cells, DNA methylation is significantly correlated with the transcript expression of these two isoforms. We show that both Mecp 2 isoforms are highly expressed in male neurons compared to male astrocytes, with Mecp2e1 expressed at higher levels than Mecp2e2. Our data indicate that higher DNA methylation at the Mecp2 regulatory element(s) is associated with lower levels of Mecp2 isoforms in male astrocytes compared to male neurons.
\end{abstract}

Keywords: Rett syndrome; neurodevelopmental disorders; DNA methylation; epigenetics; MeCP2 isoforms; neurons; astrocytes; brain cells

\section{Introduction}

The X-linked methyl CpG binding protein 2 (MECP2/Mecp2) gene encodes for two MeCP2 protein isoforms, E1 and E2. MeCP2 is a major transcriptional regulator in the brain with solid links to neurodevelopmental disorders [1]. Altered expression and function of $M E C P 2 / M e c p 2 / \mathrm{MeCP} 2$ have been linked to Rett syndrome (RTT) [2-4], MECP2 duplication syndrome (MDS) [5-7], autism spectrum disorders (ASD) [8,9], and fetal alcohol spectrum disorders (FASD) [10,11]. MeCP2-associated diseases have a strong sex correlation (especially in the case of RTT and MDS), possibly due to the X-linked nature of the $M E C P 2$ gene [12] and/or X chromosome inactivation (XCI) in females [13]. Currently, these disorders have no cure, and restoring the normal MeCP2 levels is suggested as a possible therapeutic approach. Therefore, a better understanding of Mecp2/MeCP2 regulation in brain cells is critically 
important. Here, we aimed at studying the sex-specific levels of Mecp 2 isoforms in the two major cell types of the brain, namely neurons and astrocytes.

Originally, research on MeCP2 was mostly focused in neurons, primarily due to the belief that symptoms resulting from Mecp 2 loss of function were of neuronal origin. However, the discovery of MeCP2 expression in glia, and the functional consequences of glial Mecp 2 loss on neuronal phenotypes, caused a paradigm shift in the MeCP2 field [14]. Independent groups, including our team, have shown that total Mecp2/MeCP2 and MeCP2E1 expression are higher in neurons than in astrocytes $[15,16]$. We have shown differential expression of Mecp 2 isoforms during neural stem cell differentiation $[17,18]$ and in the adult brain regions that have different cellular composition [19]. However, it is still unclear whether the expression of Mecp 2 isoforms in embryonic brain cell types is sex-dependent.

We have characterized DNA methylation of six regulatory elements (REs) in the Mecp2 promoter (R1-R3) and intron 1 (R4-R6) that may impact Mecp2 isoform-specific expression [17]. DNA methylation at these REs was correlated with Mecp2 isoforms in different adult mouse brain regions [19], the embryonic forebrain [20], and alcohol-mediated change in Mecp 2 isoforms [18]. Hypermethylation of the MECP2 promoter has also been detected in ASD patients with reduced MECP2 levels [8,9], where there is a higher sex-association towards males ( 3 to 4 times higher in males compared to females) [21,22]. Therefore, correlational studies of DNA methylation at the Mecp2 REs with Mecp2 isoform-specific expression were done in male brain cells. Our results highlight the existence of a cell type-specific DNA methylation pattern at the Mecp2 gene locus in male neurons and male astrocytes.

\section{Results}

\subsection{Establishment of Sex-Specific Cultures of Male and Female Primary Neurons and Astrocytes}

To characterize the cell type-specific expression of Mecp 2 isoforms in both sexes, we isolated and cultured male and female primary embryonic cortical neurons and astrocytes according to our previously established and validated protocols $[15,23,24]$. Separation and confirmation of embryonic sexes were performed by independent and parallel techniques. First, the male and female embryos were separated based on visual observation of testes in male embryos and fallopian tubes and ovaries in female embryos, respectively (Figure 1A). The sex of cultured cells was further confirmed by qRT-PCR-based detection of Xist transcripts, which are specific to females [25]. As expected, Xist transcripts were absent in male cells and were exclusively detected in female cells (Figure 1B). PCR-based detection of Il3/Sry genes was used to confirm the sex of male cells. Sry is expressed in male cells [26], while Il3 is expressed in male and female cells [27]. Male cells showed detection of both Sry and Il3, thereby confirming the male origin of these cells (Figure 1C).
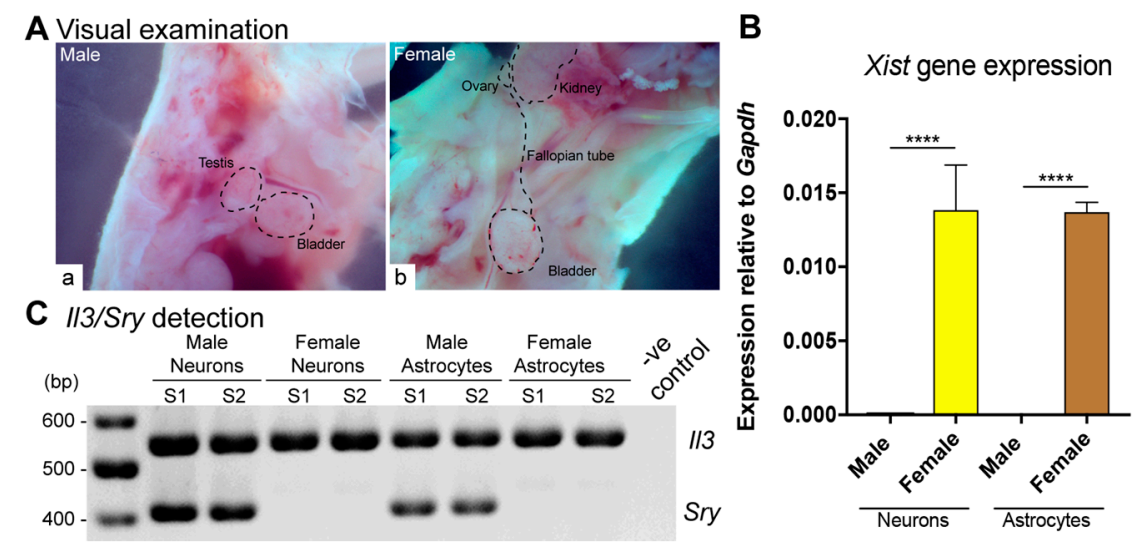

Figure 1. Culturing sex-specific neurons and astrocytes and their molecular identification. (A) Visual examination-based separation of male (a) and female (b) embryos based on observation of testes and ovaries, respectively. (B) Confirmation of sex-specific nature of cultured neurons and astrocytes using female-specific Xist gene expression. Xist transcript expression is detected only in female cells. For all 
samples, $N=3$, except for female neurons, for which $N=2$. Error bars represent standard error of the mean (SEM) with **** $p<0.0001$. (C) Il3/Sry-based confirmation of male or female sex using agarose gel electrophoresis. PCR product sizes are Sry, 402 bp; Il3, 544 bp. Sry is detected only in males, while Il3 is detected in all samples.

\subsection{Mecp2 Isoforms Show Cell Type- and Sex-Specific Expression in Neurons and Astrocytes}

Mecp2/MeCP2 expression level in brain cells is cell type-dependent $[15,16]$. Mecp2 is X-linked and undergoes XCI escape with biallelic expression as early as the two-cell stage of mouse embryonic development [28]. Therefore, we determined Mecp $2 e 1$ and Mecp $2 e 2$ transcript levels in male neurons and male astrocytes and compared these levels to female cells (Figure 2).

A

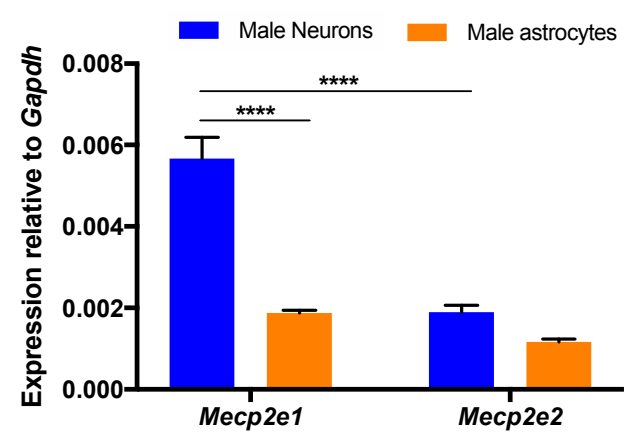

B

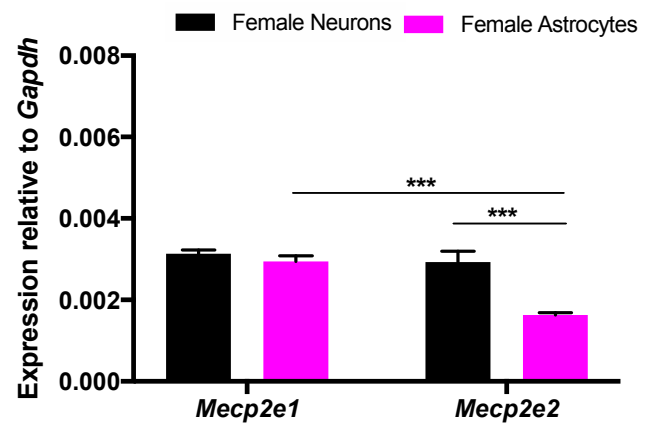

Figure 2. Cell type- and sex-specific expression of Mecp2 isoforms in primary mouse embryonic neurons and astrocytes. The figure illustrates cell type-specific and sex-specific comparison of Mecp2 isoforms in neurons and astrocytes. (A) Mecp2 expression in male neurons and astrocytes. (B) Mecp2 expression in female neurons and astrocytes. Significant differences are indicated with ${ }^{* * * *} p<0.0001$, ${ }^{* * *} p<0.001$. For male neurons, male astrocytes, and female astrocytes, $N=3 \pm$ SEM and $N=12 \pm$ SEM (biological replicates are reported as $N$, while technical repeats of biological replicates are reported as $N)$. For female neurons, $N=2 \pm$ SEM and $N=8 \pm$ SEM. The transcript levels were normalized to the endogenous control Gapdh.

Cell type-specific expression of Mecp2e1 was 3.02-fold $(p<0.0001)$ higher in male neurons compared to male astrocytes (Figure 2A). Similarly, Mecp2e2 transcripts were 1.63-fold higher in male neurons than in male astrocytes. This suggested that both Mecp 2 isoforms have higher expression in male neurons compared to male astrocytes. This pattern was not seen in female cells, and Mecp $2 e 1$ transcript levels were similar for female neurons and astrocytes (1.06-fold: female neurons vs. female astrocytes). However, Mecp2e2 transcript levels were higher (1.80-fold) in female neurons in contrast to female astrocytes ( $p<0.001$; Figure 2B). In male neurons, Mecp2e1 transcripts were higher than Mecp2e2 (2.99-fold, $p<0.0001$; Figure 2A). Similarly, in male astrocytes, Mecp2e1 levels were slightly higher than Mecp2e2 (1.61-fold). In female neurons, Mecp2e1 and Mecp2e2 levels were similar (1.07-fold; Figure 2B), but Mecp2e1 levels were higher than Mecp2e2 in female astrocytes (1.80-fold, $p<0.001)$. Therefore, in both cell types, except for the female neurons, Mecp2e1 transcripts were the major transcripts.

These observations provide evidence that these two studied brain cell types (neurons and astrocytes) have differential expression of Mecp 2 isoforms depending on the sex of embryos, raising the possibility that the transcription levels of these two isoforms are differentially regulated.

2.3. DNA Methylation at the Mecp2 Regulatory Elements May Contribute to Higher Expression of Mecp2 in Male Neurons Compared to Male Astrocytes with Lower Mecp2 Expression

In a previous section, we showed the higher transcript levels of Mecp2 isoforms in male neurons compared to male astrocytes, which was highly significant for Mecp2e1. To study if DNA methylation 
impacts the detected difference in Mecp2e1 and Mecp2e2 transcript levels, we analyzed male neurons and male astrocytes that contain only one X-chromosome, avoiding complications of X-linked inactivation that exists in female cells. For these studies, we analyzed DNA methylation patterns at the known Mecp2 REs [17,18] (Figure 3B). Previous genome-wide DNA methylation analyses have indicated differential DNA methylation at the CpG islands that include the CpG islands, $\mathrm{N}$ shore, $\mathrm{N}$ shelf, $\mathrm{S}$ shore, and $\mathrm{S}$ shelf that flank the CpG islands (Figure 3A,B). The 'shores' comprise the 2-kb region flanking CpG islands and display dynamic DNA methylation patterns in contrast to the lower DNA methylation levels seen at CPG islands. The 'shelves' are located $4 \mathrm{~kb}$ further away from the CpG islands. Similar to shores, these regions are differentially methylated [29-31].

These previous studies suggest that hypomethylated or hypermethylated blocks of CpGs such as $\mathrm{CpG}$ islands or $\mathrm{CpG}$ shores may have a significant impact in determining the diagnosis of disease conditions such as cancer [31-33]. Also, increased methylation of the $M E C P 2$ promoter in autistic male patients is reported to correlate with reduced $M E C P 2$ expression [8]. Therefore, we analyzed DNA methylation at individual CpG sites (Figure 3C,D) and average DNA methylation over the entire REs (Figure 4). As reported previously [17,18], Mecp2 promoter regions R1, R2, and R3 contained 13, 4, and 2 CpG sites, respectively. The intron 1 silencer element contained three REs, namely R4, R5, and R6, with 1, 1, and 2 CpG sites, respectively (Figure 3B).

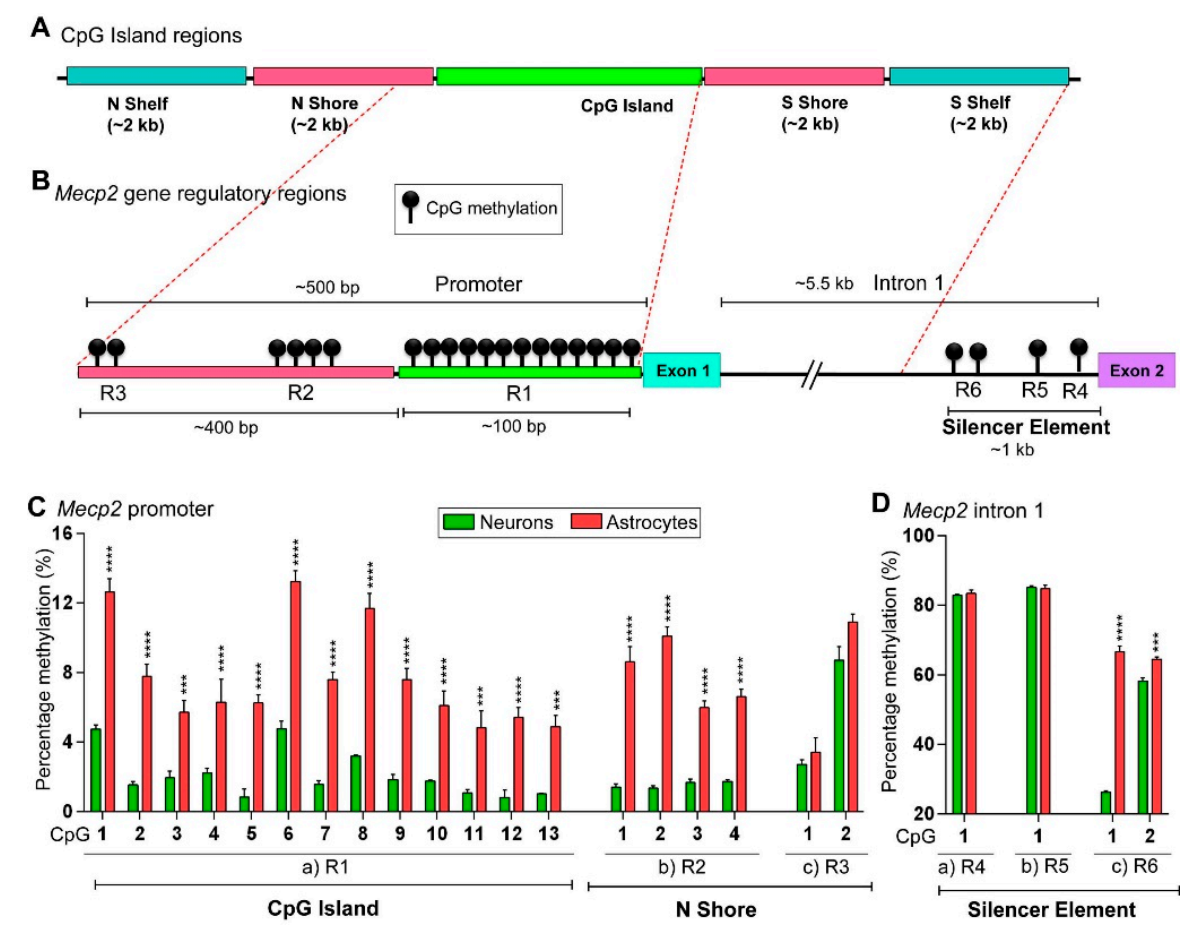

Figure 3. Bisulfite pyrosequencing analysis of DNA methylation at the Mecp2 regulatory elements (REs) in male neurons and male astrocytes. (A) Schematic representation of $\mathrm{CpG}$ island regions characterized based on distribution of CpGs. Part A of the figure is modified from [34,35] (not drawn to scale). (B) Location of CpGs in Mecp2 gene REs. Figure not drawn to scale. The figure illustrates the six previously reported REs [17], R1-R3 promoter Res, and R4-R6 intron 1 silencer element REs (not drawn to scale). The methylation symbol indicates the number of CpGs found within each RE: R1: 13 CpGs, R2: 4 CpGs, R3: 3 CpGs, R4: 1 CpG, R5: 1 CpG, and R6: 2 CpGs. (C) Percentage of DNA methylation (\%) of CpG sites within the Mecp2 promoter regions (a) R1, (b) R2, and (c) R3. (D) Percentage of DNA methylation (\%) of CpG sites within the Mecp 2 intron 1 regions (a) R4, (b) R5, and (c) R6. Significant differences between male neurons and male astrocytes are indicated with ${ }^{* * * *} p<0.0001,{ }^{* * *} p<0.001$, $N=3 \pm$ SEM. 


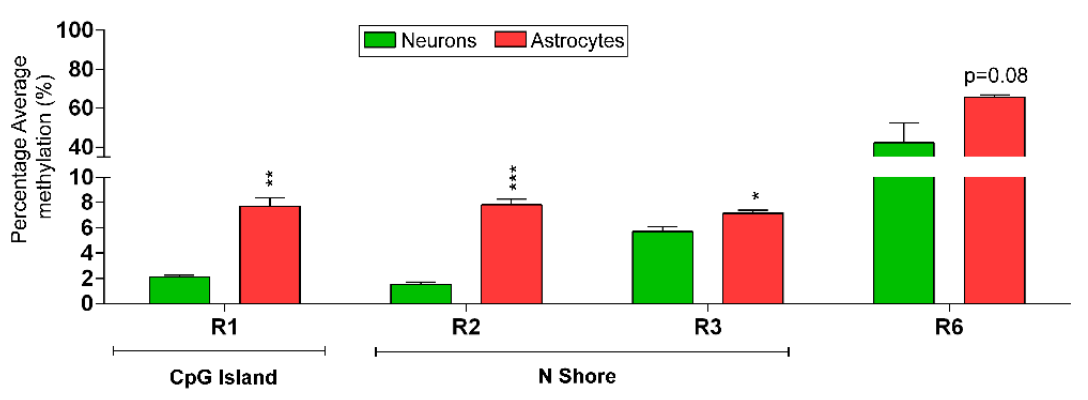

Figure 4. Average DNA methylation patterns over the Mecp2 regulatory elements (REs) in male neurons and male astrocytes. Average percentage DNA methylation of all CpG sites in the promoter regions R1 (13 CpGs), R2 (4 CpGs), R3 (2 CpGs), and R6 of the intron 1 region (2 CpGs), in male neurons and male astrocytes. Significant difference between male neurons and male astrocytes is indicated with ${ }^{* * *} p<0.001,{ }^{* *} p<0.01$, or ${ }^{*} p<0.05 . N=3$. Error bars indicate standard error of the mean (SEM).

Based on a general repressive role of DNA methylation, we hypothesized that higher Mecp2 expression in male neurons is associated with lower DNA methylation at the Mecp2 REs, while lower Mecp 2 expression in male astrocytes is associated with higher DNA methylation. First, we assigned the CpG island regions illustrated in Figure 3A to Mecp 2 gene REs (Figure 3B). R1 of Mecp 2 promoter is considered as a portion of a CpG island, which spans over exon 1 . On the other hand, R2 and R3 belong to the $\mathrm{N}$ shore (Figure $3 \mathrm{~B}$ ). As intron $1 \mathrm{REs}$ are located more than $4 \mathrm{~kb}$ downstream of the $\mathrm{CpG}$ island, they do not belong to either an $\mathrm{S}$ shelf or $\mathrm{S}$ shore. Based on previous studies in autistic patients and a rare case of an RTT patient $[8,9,36]$, we hypothesized that blocks of DNA methylation at the CpG island and/or N shore may have differentially methylated CpG blocks, which may contribute to differential Mecp 2 expression in male neurons and male astrocytes. Bisulfite pyrosequencing experiments showed that the percentage of DNA methylation at the $13 \mathrm{CpGs}$ within the Mecp 2 promoter region $\mathrm{R} 1$ was higher in male astrocytes compared to male neurons (CpG1: $+7.87 \%$, CpG2: $+6.23 \%$, CpG3: $+3.77 \%$, CpG4: $+4.08 \%$, CpG5: $+5.42 \%$, CpG6: $+8.46 \%$, CpG7: $+6.02 \%$, CpG8: $+8.49 \%$, CpG9: $+5.75 \%$, CpG10: $+4.33 \%$, CpG11: $+3.76 \%$, CpG12: $+4.60 \%$, and CpG13: $+3.86 \%$ ) (Figure 3C). In general, DNA methylation $(5 \mathrm{mC})$ of regulatory elements is associated with decreased gene transcription; therefore, higher DNA methylation could lead to reduced gene expression [37]. Our results are consistent with this idea that increased DNA methylation at these $\mathrm{CpG}$ dinucleotides in male astrocytes may collectively contribute to the lower Mecp2 levels in these cells. Similarly, the percentage of DNA methylation at the four CpG sites at the R2 region was significantly higher in male astrocytes compared to male neurons (CpG1: $+7.22 \%$, CpG2: $+8.74 \%$, CpG3: $+4.32 \%$, and CpG4: $+4.88 \%$ ). However, DNA methylation at R3 CpGs was not statistically significant (CpG1: $+0.71 \%$ and CpG2: $+2.18 \%$ ). Regardless, the proximal Mecp 2 promoter, which contains R1 and R2, showed a higher percentage of DNA methylation in male astrocytes compared to male neurons, potentially contributing to the lower Mecp2e1 and Mecp2e2 levels in male astrocytes. These data imply that in contrast to R1 and R2, DNA methylation at R3 of the promoter may not play a significant role in differential expression of Mecp2 isoforms in male neurons and male astrocytes.

We then studied the Mecp2 intron 1 regions for DNA methylation changes (Figure 3D). The CpG sites of R4 and R5 were similarly methylated in male astrocytes and male neurons (difference: CpG1-R4: $+0.54 \%$, CpG1-R5: $-0.36 \%$ ). However, the two CpGs within R6 had a significantly higher percentage of DNA methylation in male astrocytes compared to male neurons (CpG1: $+40.40 \%$ and CpG2: $+6.29 \%$ ). Since this DNA methylation pattern is in agreement with our previous studies highlighting their role in Mecp 2 regulation [17,18], it is likely that higher methylation at the R6 region may contribute to lower expression of Mecp 2 isoforms in male astrocytes compared to male neurons. It is possible that DNA methylation at the intronic R4 and R5 may not play significant roles in differential expression of Mecp2 isoforms in male neurons and male astrocytes. 
Furthermore, we calculated the average DNA methylation of the REs that contained more than one CpG site (R1, R2, R3, and R6). Both R1 (+5.59\%) and R2 (+6.29\%) showed greater than 5\% DNA methylation change over the entire region (Figure 4).

Although individual CpG sites of R3 were not significantly changed, the average DNA methylation over promoter R3 was slightly higher $(+1.44 \%, p<0.05)$ in male astrocytes. The average DNA methylation over individual R4-R6 CpG sites was not statistically significant. However, R6 showed $+23.34 \%(p=0.08)$ higher average DNA methylation in male astrocytes than in male neurons. Also, average methylation over the intron 1 silencer element (R4-R6) showed $+11.45 \%$ hypermethylation in male astrocytes compared to male neurons. In other words, the difference in average DNA methylation between male neurons and male astrocytes was higher in the CpG island (R1), while the difference was reduced further away from the exonic region (R3). However, the average methylation within the intron 1 REs was similar between the two cell types, implying that the distinct differential average methylation observed in CPG island regions was not significantly different in the analyzed R4-R6 intronic region. Overall, these analyses showed that male neurons have significantly lower DNA methylation, mainly at the Mecp 2 promoter REs, in contrast to male astrocytes. DNA methylation levels at the Mecp 2 REs may contribute to the epigenetic regulation of Mecp2 isoforms in male neurons and male astrocytes.

\subsection{Expression of Mecp2 Isoforms Correlates with DNA Methylation at the Mecp2 REs}

To gain insight into the impact of DNA methylation on Mecp2e1 and Mecp2e2 expression in male neurons and astrocytes, we performed a correlational analysis between Mecp 2 isoform-specific transcripts and DNA methylation at the individual CpG sites of R1-R6 (Figure 5). First, we determined the relation between Mecp2e1 transcripts in male neurons with Mecp 2 promoter regions (Figure 5A). For R1, CpG1 showed a very strong negative correlation with Mecp2e1 expression $(r=-0.99, p<0.05)$, implicating a repressive role of DNA methylation at this CpG. Similarly, CpG8 showed a very strong negative correlation with Mecp2e1 $(r=-0.99, p<0.05)$. Although CpG13 showed a negative correlation with Mecp2e1, it was not statistically significant $(r=-0.97, p=0.15)$. The other CpG sites (CpG 2, 3, 4, $5,6,7,9,10,11$, and 12) showed varying degrees of positive correlations that were not statistically significant. Correlations between DNA methylation at the CpG sites of promoter R2 and Mecp2e1 expression had a very strong positive nature $(r>-0.9)$. CpG3 and CpG4 showed significant positive correlation (CpG3: $r=+0.99, p<0.05$; CpG4: $r=+0.99, p=0.051$ ), usually referring to an active role. Mecp2e1 showed a positive but statistically insignificant correlation with DNA methylation at the promoter R3 and at the intron 1 regions R3, R4, and R6.

When correlation between Mecp2e2 and DNA methylation at the REs was studied, we found that Mecp2 REs showed varying degrees of positive and negative correlations. However, these correlations were statistically insignificant. It should be noted that this might not necessarily suggest the absence of a role for DNA methylation in Mecp2e2 regulation in male neurons. This may be due to the involvement of different types of DNA methylation or that DNA methylation does not play a significant role here.

Unlike male neurons, which showed widely distributed correlation patterns between DNA methylation and Mecp 2 transcripts; male astrocytes demonstrated distinct DNA methylation patterns that correlated with Mecp2 expression (Figure 5B). Both Mecp2e1 and Mecp2e2 expression in male astrocytes negatively correlated with DNA methylation of all CpGs of R1, which is in agreement with the repressive role of DNA methylation at R1 that we previously noted [17,18]. Among them, correlation between Mecp2e1 and R1: CpG2 $(r=-0.9984, p<0.05)$ and R1: CpG3 $(r=-0.9994$, $p<0.05)$ was significant. Similarly, Mecp2e2 showed a negative and significant correlation with DNA methylation at R1: CpG7 $(r=-0.9999, p<0.01)$. These data suggest that R1 may play a repressive role for both Mecp2 isoforms. Correlation between DNA methylation at R2, R3, and intron 1 R4-R6 regions and Mecp2 isoforms was statistically insignificant. However, R2: CpG1, R2: CpG2, and R3: CpG1 showed a positive correlation with both Mecp2 isoforms. In contrast, R2: CpG3, R2: CpG4, and R3: CpG2 showed a negative correlation with both Mecp2 isoforms. These data suggest that DNA methylation plays a different role in regulating Mecp 2 isoform-specific expression in male astrocytes 
and male neurons. Similar to neurons, DNA methylation at R4-R6 showed a positive but statistically insignificant correlation with Mecp2e1. Correlation between Mecp2e2 and DNA methylation at R4-R6 was not fully consistent between the two cell types.
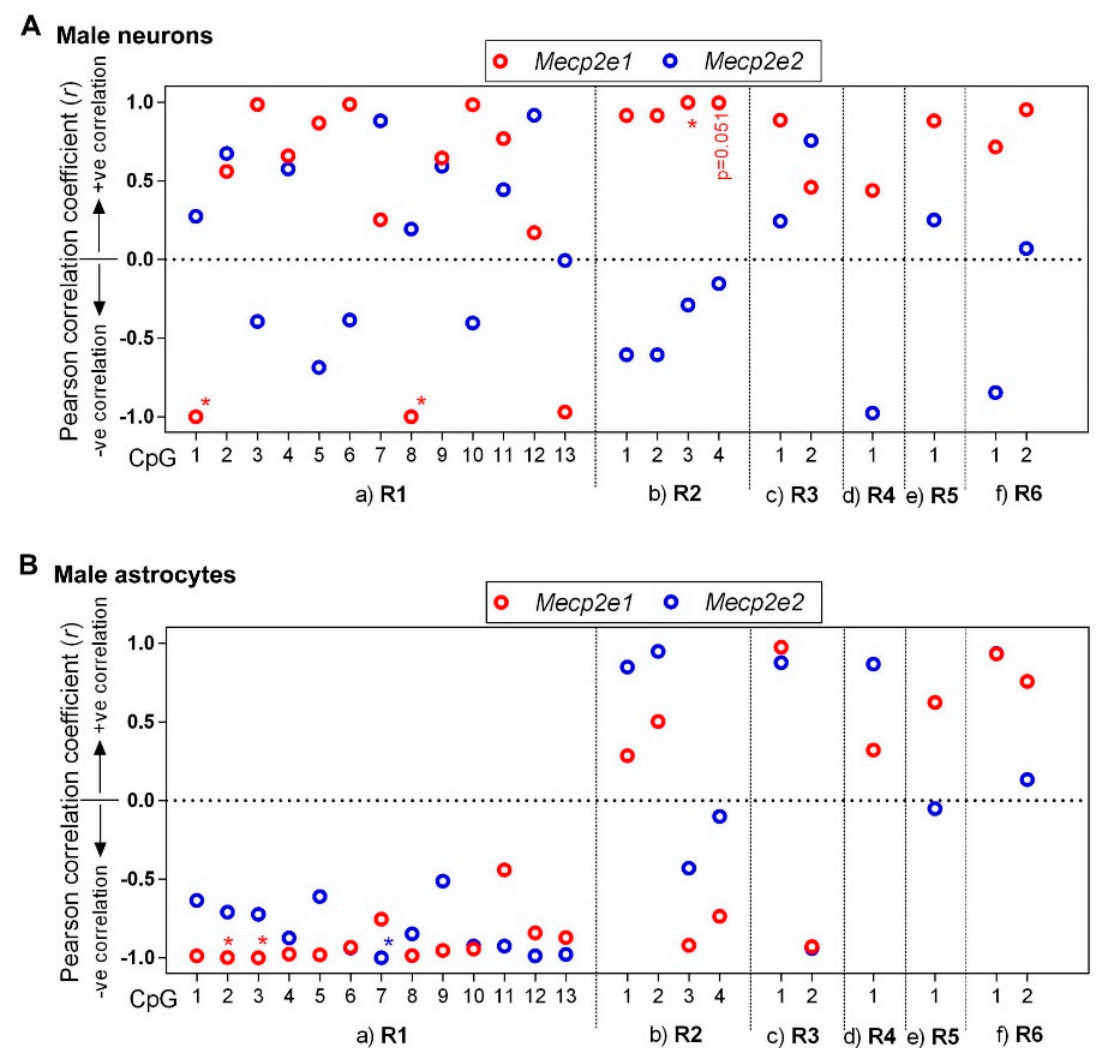

Figure 5. Correlation analyses between DNA methylation at the Mecp2 regulatory elements (REs) and Mecp2 isoform-specific expression in male neurons and male astrocytes. (A) Male neurons; (B) male astrocytes. All graphs represent the Pearson correlation coefficient ( $r$ ) for Mecp $2 e 1$ (red) and Mecp2e2 (blue). Statistical significance of the correlation is indicated by ${ }^{*} p<0.05 ; N=3$. Individual CpG sites at the promoter regions (a) R1, (b) R2, and (c) R3 and intron 1 regions (d) R4, (e) R5, and (f) R6 are shown on the $x$-axis.

\section{Discussion}

Understanding how Mecp2/MeCP2 expression is controlled in major brain cell types is important not only developmentally, but also for human diseases affecting the brain. Such studies would provide insight in understanding how different levels of Mecp2/MeCP2 isoforms contribute to the maintenance of proper brain cellular functions and activities. Accordingly, investigation of Mecp 2 cell type-specific expression and regulation may provide valuable insights on how regulatory mechanisms can be used for potential strategies for MeCP2-associated diseases. Therefore, our study contributes to cell typeand sex-specific health research in brain cells.

Our study explored the expression of Mecp 2 isoforms in a sex-specific manner in primary neurons and astrocytes. The sex-specific difference in the basal level of Mecp 2 transcripts in embryonic brain cells highlights the importance of considering sex as a biological factor in MeCP2-associated neurodevelopmental disorders. In agreement with sex-specific levels of MeCP2, it is the study of Mecp2/MeCP2 expression in male and female rat brains during development [38] that indicated higher Mecp2/MeCP2 levels in the female amygdala and ventromedial hypothalamus at postnatal day 1 (P1) compared to male brain regions. However, at P10, males expressed more Mecp2/MeCP2 in the preoptic area. The observed higher expression of Mecp 2 in females than males in the rat brain at P1 is in agreement with the higher Mecp2e1 levels in astrocytes and Mecp2e2 levels in neurons and 
astrocytes. However, our study shows that in E18-isolated primary brain cells, Mecp2e1 expression is higher in male than female neurons. In agreement with a previous report [38], our study demonstrates the possibility that Mecp 2 expression is sex-dependent, cell type-specific, and isoform-specific. These results may also implicate age/developmental stage-specific regulatory mechanisms.

Several studies have shown XCI escape and biallelic expression of the X-linked genes such as histone deacetylase 6 (Hdac6) and lysine demethylase 6A (Kdm6a) that are associated with the more accessible chromatin structure at the promoters of these genes, as indicated by DNase I hypersensitivity, occupancy of RNA polymerase phosphorylated at Ser 5 (PolII-S5p), recruitment of CCCTC-binding factor (CTCF), and enrichment of active histone modifications such as H3K4me3 [39,40]. Not only $M E C P 2$, but also other X-linked genes, such as GRB2-associated-binding protein 3 (GAB3), ribosomal protein S4, X-linked (RPS4X), Jumonji/ARID domain-containing protein 1C (JARID1C), ubiquitin-like modifier activating enzyme 1 (UBE1), baculoviral IAP repeat-containing protein 4 (BIRC4), and solute carrier family 16 member 2 (SLC16A2), show differential expression between the two sexes, with higher expression in female bovine fetal muscle tissues compared to males [41]. Generally, when genes escape XCI in females, they show higher expression in females due to biallelic expression, in contrast to monoallelic expression in males. However, here, the higher expression in females was not observed in all cases, suggesting such discordance regarding XCI escape could be due to other complicated regulatory mechanisms. Literature supports such discordance where male cells show higher gene expression. For instance, using microarray analysis, Talebizadeh et al. [42] demonstrated that only $10 \%$ of the $299 \mathrm{X}$-linked genes that they studied showed higher expression levels in the female human cerebrum (female (F) /male (M) ratio $\geq 0.7$ ), and $17 \%$ of the genes showed higher $\mathrm{X}$-linked gene expression in the male cerebrum ( $\mathrm{F} / \mathrm{M}$ ratio $\geq 1.5$ ) [42]. The $M E C P 2$ uni-gene cluster (Hs.200716) showed an F/M ratio $=0.8$ in the cerebrum in this study, suggesting $M E C P 2$ abundance in the male cerebellum may be higher than in the female cerebellum. The cerebellum is a brain region with the highest neuronal density in the brain $[43,44]$. Therefore, it is worth questioning whether there are deviating or complex Mecp 2 regulatory mechanisms seen in neurons, and more specifically, in male neurons, which drive higher Mecp 2 expression levels. However, in our study, the two Mecp2 isoforms showed opposing trends (Mecp2e1: male > female; Mecp2e2: male < female). The presence of correlation between Mecp2e1 and DNA methylation and the absence of such correlation with Mecp2e2 sought us to speculate whether DNA methylation plays a role in this observation. It is also possible that an alternative splicing mechanism is shifted in male neurons in contrast to female neurons. In general, DNA methylation can regulate alternative splicing [10]. In our previous studies in differentiating murine neural stem cells, we proposed that DNA methylation status at R1 (promoter), R4, and R5 (intron 1 silencer element) might be involved in alternative splicing of the Mecp2 gene. The potential impact of DNA methylation at the Mecp2 intron 1 silencer element in regulating Mecp2 splicing in neurons and astrocytes is also a possibility. However, investigating the role of DNA methylation in regulating Mecp 2 alternative splicing was not within the scope of this current study. Regardless, it is important to determine the role of DNA methylation in Mecp2 alternative splicing, as this may modulate the expression of Mecp2e1 and Mecp2e2 in different type of brain cells including neurons and astrocytes. It is possible that a 'kinetic coupling model' of cotranscriptional splicing via DNA methylation- and CTCF-mediated mechanisms [45] may contribute to Mecp2 splicing in neurons and astrocytes. Regardless, establishing the precise role of DNA methylation in Mecp2 splicing warrants further investigations.

Here, DNA methylation at the six Mecp2 REs was determined by bisulfite pyrosequencing, followed by correlational analysis in male neurons and male astrocytes. While comparison between male neurons and male astrocytes highlighted a negative correlation between Mecp 2 expression and DNA methylation, correlational analysis in male neurons showed otherwise for Mecp 2 promoter R2. Generally, bisulfite pyrosequencing does not distinguish between $5 \mathrm{mC}$ and $5 \mathrm{hmC}$ DNA methylation marks [46] and may somehow overrepresent $5 \mathrm{mC}$ [47]. Therefore, additional analysis of $5 \mathrm{mC}$ and $5 \mathrm{hmC}$ at the Mecp2 REs may provide further insight. Within the body, the brain and specifically 
neurons show the highest conserved non-CpG DNA methylation [48], which could provide more in-depth knowledge. We observed that the strongest correlation to be in the R1 promoter region between male neurons and male astrocytes, which was relatively small in terms of the percentage of DNA methylation. However, as we reported for Mecp 2 in differentiating brain-derived embryonic neural stem cells, even small percentage changes at its REs might be biologically significant and cause 2-3-fold change at the MeCP2 protein levels [17]. In general, DNA methylation may also exist in the form of $\mathrm{CpH}$ (rather than $\mathrm{CpG}$ ) and may be differently involved in primary neurons and astrocytes. Although $\mathrm{CpH}$ DNA methylation was not part of our reported study here, investigation of $\mathrm{CpH}$ DNA methylation would be important in the context of cell type-specific expression of Mecp2e1/e2.

Previously, we reported differential correlation of Mecp2 isoforms with DNA methylation that varied with the stages of neural stem cell differentiation [17] or between murine strains [20]. For differentiating neural stem cells isolated from E14 mouse embryos, the correlation was seen in almost all the CpG sites with both Mecp2 isoforms. However, in in vivo differentiated neurons isolated from E18 embryos in this study, significant correlation was limited to four CpGs in the Mecp 2 promoter and with Mecp2e1. Similarly, the number of significant correlations observed in male astrocytes isolated from E18 embryos was low, albeit the correlations that were observed for both Mecp 2 isoforms. This may implicate that DNA methylation might be more critical at earlier stages of embryonic development and during neural stem cell differentiation, in comparison to differentiated cell types at later stages of embryonic development. These differences further emphasize the existence of cell type-specific Mecp 2 regulatory mechanisms. Yet, differential cell type-specific expression and/or regulation make it challenging to develop strategies to rescue abnormal MeCP2 expression in disease conditions. For instance, decitabine treatment during embryonic neural stem cell differentiation induced Mecp2e1/MeCP2E1 [17], while similar studies in fibroblasts isolated from RTT patients did not respond to 5-aza-2'-deoxycytidine (similar to decitabine) treatment at lower concentrations [49]. It is questionable as to whether similar treatments would be successful in elevating Mecp2/MeCP2 expression in differentiated cell types such as neurons and in later stages of development or adulthood. DNA-demethylating agents that are capable of inducing Mecp2/MECP2/MeCP2 levels may be helpful for RTT. However, it is unclear if knowledge on the effect of DNA methylation on Mecp 2 could be used in MECP2 duplication syndrome. In lens epithelial cells, scientists demonstrated that a DNMT inhibitor (which can also be a DNA-demethylating agent), zebularine, attenuated MeCP2 expression in a time- and dose-dependent manner [50]. Similarly, in the human fetal retinal pigment epithelial cells, 5-aza-2'-deoxycytidine reduced MeCP2 expression in a dose-dependent manner [51]. Therefore, it is possible that depending on the cell type, the effect of DNA-demethylating agents and/or DNMT inhibitors on MeCP2 expression may be different.

\section{Materials and Methods}

\subsection{Ethics}

Our experimental studies were performed in agreement with the standards of the Canadian Council on Animal Care with approval of the Office of Research Ethics, University of Manitoba. All procedures were reviewed in advance of conducting the experiments and were approved by the University of Manitoba Protocol Management and Review Committee at the Bannatyne Campus, under the approved protocol number 12-031/1/2 (16 June 2014) and subsequent renewal(s).

\subsection{Primary Culture of Embryonic E18.5 Neurons}

Primary embryonic neurons from the cortex of E18.5 CD1 mice were isolated and cultured as previously described $[15,23,24]$. Embryos were separated based on the sex by visual sex recognition under a dissecting Zeiss microscope. In brief, dissected cortices were dissociated by papain and trituration through a Pasteur pipette. Dissociated cells were resuspended in neurobasal media supplemented with B27 and were then plated at a density of $1.2 \times 10^{5}$ cells $/ \mathrm{mL}$ in poly-lysine-coated 
dishes. Half of the media was replaced after 72 hours (h) and was refreshed every $48 \mathrm{~h}$ thereon. According to published protocols for primary neurons, cells were collected after eight days in culture.

\subsection{Primary Culture of Embryonic E18.5 Astrocytes}

Primary embryonic astrocytes from the cortex of E18.5 CD1 mice were cultured as previously described $[15,24]$. Embryos were separated based on the sex and as described above for primary neurons. In brief, dissected cortices were further dissociated using papain enzyme and triturated with Pasteur pipette. Cells were subsequently resuspended in minimum essential medium (MEM, Thermo Fisher Scientific) supplemented with $10 \%$ FBS. Cells were then seeded at a density of $2 \times 10^{5}$ cells $/ \mathrm{mL}$ in poly-lysine-coated dishes. Media were replaced every $48 \mathrm{~h}$ until the day of collection. According to the published protocols for primary astrocytes, cells were collected after 15 days.

\subsection{Culture and Identification of Sex-Specific Neurons and Astrocytes}

Genomic DNA was extracted from neurons and astrocytes by DNeasy Blood and Tissue Kit (Qiagen). Semiquantitative polymerase chain reaction (PCR)-based amplification of sex-determining region protein gene on $\mathrm{Y}$ chromosome (Sry) was done as previously reported [17] to identify and confirm male cells. We used the interleukin 3 (Il3) gene as an autosomal gene and an internal control for both sexes. PCR products were identified based on size (Sry, 402 base pairs (bp); Il3, 544 bp). RNA was extracted from male and female cells by Trizol extraction method (Life Technologies Inc., 15596-026) and mirVana RNA extraction kit (Thermo Fisher Scientific, AM1560), respectively. Quantitative reverse transcription PCR (qRT-PCR) for X-inactive specific transcripts (Xist) was done for female cells as previously described $[17,52]$.

\subsection{Quantitative RT-PCR ( $q R T-P C R)$}

RNA was extracted from male cells and female cells by Trizol and mirVana RNA extraction kit methods, respectively. RNA that was converted to cDNA using previously established protocols prior to qRT-PCR [17]. Relative gene expression was calculated with reference to the housekeeping gene glyceraldehyde 3-phosphate dehydrogenase (Gapdh).

\subsection{DNA Methylation Analysis by Bisulfite Pyrosequencing}

Bisulfite pyrosequencing was performed for the six Mecp2 REs (R1 to R6) as a service at the Hospital for Sick Children, Toronto, Canada, as we reported previously [17-20]. For the sequence of the primers, please refer to our previous reports [17-20].

4.7. Correlation Analysis between Detected DNA Methylation at the Mecp2 Regulatory Elements and Transcript Expression Levels of Mecp2 Isoforms

Pearson's correlation analysis and linear regression were done as we reported previously $[17,19]$, and statistical significance was determined at $p<0.05$ as described elsewhere [17-19].

\subsection{Statistical Analysis}

The graphs represent an average of 2-3 independent biological experiments $(N=2-3)$ and 8-12 technical replicates $(n=8-12)$ with error bars showing standard error of the mean (SEM). Statistical significance was determined at ${ }^{* * * *} p<0.0001,{ }^{* * *} p<0.001,{ }^{* *} p<0.01$, or ${ }^{*} p<0.05$. All experiments were performed in primary cortical neurons or astrocytes isolated from 2-3 separate pregnant mothers as $N=2-3$ biological replicates $[17,18,24]$. For the comparison of cell type-specific, sex-specific, and isoform-specific expression of Mecp2 isoforms, two-way ANOVA was used. 


\section{Conclusions}

In conclusion, our studies presented here report the cell type-, sex-, and isoform-specific expression of Mecp2 isoforms in primary cortical neurons and astrocytes. Neither Mecp 2 isoform showed equal expression in male and female cell types. In comparison to male astrocytes, male neurons had higher levels of Mecp 2 transcripts. Our results provide evidence that the DNA methylation of Mecp2 regulatory elements contributes to the differential expression of Mecp 2 in male neurons and astrocytes. In male neurons, Mecp2e1 was the major isoform, with approximately three-fold higher levels than Mecp2e2. The reduced methylation of the Mecp 2 response elements in male neurons may alter transcription elongation rates and splicing of the Mecp 2 transcripts, giving rise to greater levels of Mecp $2 e 1$ transcripts. The major findings of our study and potential mechanisms are summarized in Figure 6.

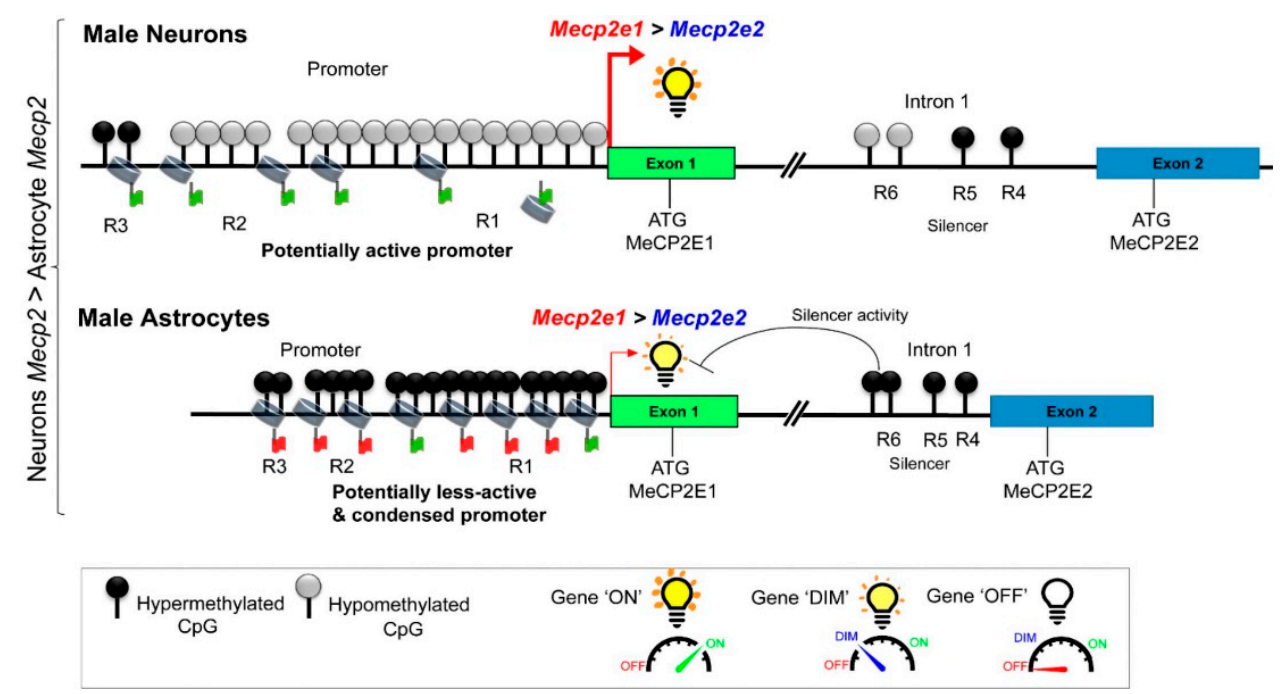

Figure 6. Proposed model for potential control of Mecp2e1 and Mecp 2e2 by DNA methylation in male neurons and male astrocytes. Based on the findings of this study, this figure illustrates a summary of the proposed mechanism of Mecp 2 regulation by DNA methylation in male neurons and male astrocytes. Higher Mecp 2 expression levels in male neurons compared to male astrocytes could be in part driven by lower DNA methylation. It is possible that a lower DNA methylation at the Mecp2 promoter in male neurons may induce an "active chromatin conformation", allowing higher transcription. In contrast, higher DNA methylation at the Mecp2 promoter in male astrocytes may cause a "less active and more condensed chromatin conformation", leading to lower Mecp2 level. We propose that DNA methylation may act as a switch for fine-tuning of the Mecp2 gene expression from "ON" (active promoter) to "DIM" (less active promoter) to "OFF" (repressed promoter).

Author Contributions: V.R.B.L., C.O.O., R.M.Z., and M.R. designed experiments. C.O.O. performed embryonic dissections and separation of embryos based on sex, imaging sex-specific embryos, RNA extraction from male cells, DNA extraction from male cells and preparing samples for bisulfite pyrosequencing. V.R.B.L. performed molecular analysis of male-female cells, extracted RNA from female cells, performed RT-PCR, performed bisulfite pyrosequencing analysis and correlational studies. R.M.Z. and M.R. performed primary neurons and astrocytes isolation and culture. J.R.D. provided scientific insight. M.R. provided conception and design, contributed reagents, materials, analysis tools, and research facilities. All authors have read and approved the final version of the manuscript.

Funding: This work is supported by funding from the Natural Sciences and Engineering Research Council of Canada (NSERC) Discovery Grant 2016-06035 to M.R., International Rett Syndrome Foundation (IRSF) grant \#3212 to M.R., and Ontario Rett Syndrome Association to M.R.

Conflicts of Interest: The authors declare no conflict of interest. 


\section{Abbreviations}

\begin{tabular}{|c|c|}
\hline $5 \mathrm{hmC}$ & 5-hydrocymethylcytosine \\
\hline $5 \mathrm{mC}$ & 5-methylcytosine \\
\hline ASD & autism spectrum disorder \\
\hline BIRC4 & baculoviral IAP repeat-containing protein 4 \\
\hline $\mathrm{bp}$ & base pairs \\
\hline cDNA & complementary DNA \\
\hline CTCF & CCCTC-binding factor \\
\hline DNMT & DNA methyltransferase \\
\hline $\mathrm{E}$ & embryonic day \\
\hline ENCODE & Encyclopedia of DNA Elements \\
\hline $\mathrm{F}$ & female \\
\hline FASD & fetal alcohol spectrum disorder \\
\hline FBS & fetal bovine serum \\
\hline GAB3 & GRB2-associated-binding protein 3 \\
\hline Gapdh & glyceraldehyde 3-phosphate dehydrogenase \\
\hline $\mathrm{h}$ & hours \\
\hline $\mathrm{Hdac}$ & Histone deacetylase 6 \\
\hline Il3 & interleukin 3 \\
\hline JARID1C & Jumonji/ARID domain-containing protein $1 \mathrm{C}$ \\
\hline$K d m 6 a$ & lysine demethylase $6 \mathrm{~A}$ \\
\hline M & male \\
\hline MDS & MECP2 duplication syndrome \\
\hline $\mathrm{MeCP} 2$ & Methyl CpG binding protein 2 \\
\hline $\mathrm{P}$ & postnatal day \\
\hline PCR & polymerase chain reaction \\
\hline PolII-S5p & RNA polymerase phosphorylated at Ser 5 \\
\hline qRT-PCR & quantitative reverse transcription PCR \\
\hline $\mathrm{R}$ & region \\
\hline $\mathrm{r}$ & Pearson's correlation coefficient \\
\hline REs & regulatory elements \\
\hline RPS4X & ribosomal protein S4 X-linked \\
\hline RTT & Rett syndrome \\
\hline SEM & standard error of the mean \\
\hline SLC16A2 & solute carrier family 16 member 2 \\
\hline Sry & sex-determining region protein gene on the $\mathrm{Y}$ chromosome \\
\hline UBE1 & ubiquitin-like modifier activating enzyme 1 \\
\hline XCI & $\mathrm{X}$ chromosome inactivation \\
\hline Xist & X-inactive specific transcript \\
\hline
\end{tabular}

\section{References}

1. Zachariah, R.M.; Rastegar, M. Linking epigenetics to human disease and Rett syndrome: The emerging novel and challenging concepts in MeCP2 research. Neural Plast. 2012, 2012, 415825. [CrossRef] [PubMed]

2. Wan, M.; Lee, S.S.; Zhang, X.; Houwink-Manville, I.; Song, H.R.; Amir, R.E.; Budden, S.; Naidu, S.; Pereira, J.L.; Lo, I.F.; et al. Rett syndrome and beyond: Recurrent spontaneous and familial MECP2 mutations at CpG hotspots. Am. J. Hum. Genet. 1999, 65, 1520-1529. [CrossRef] [PubMed]

3. Amir, R.E.; Van den Veyver, I.B.; Wan, M.; Tran, C.Q.; Francke, U.; Zoghbi, H.Y. Rett syndrome is caused by mutations in X-linked MECP2, encoding methyl-CpG-binding protein 2. Nat. Genet. 1999, 23, $185-188$. [CrossRef] [PubMed]

4. Olson, C.O.; Pejhan, S.; Kroft, D.; Sheikholeslami, K.; Fuss, D.; Buist, M.; Ali Sher, A.; Del Bigio, M.R.; Sztainberg, Y.; Siu, V.M.; et al. MECP2 Mutation Interrupts Nucleolin-mTOR-P70S6K Signaling in Rett Syndrome Patients. Front. Genet. 2018, 9, 635. [CrossRef] 
5. Meins, M.; Lehmann, J.; Gerresheim, F.; Herchenbach, J.; Hagedorn, M.; Hameister, K.; Epplen, J.T. Submicroscopic duplication in Xq28 causes increased expression of the MECP2 gene in a boy with severe mental retardation and features of Rett syndrome. J. Med. Genet. 2005, 42, e12. [CrossRef]

6. Ariani, F.; Mari, F.; Pescucci, C.; Longo, I.; Bruttini, M.; Meloni, I.; Hayek, G.; Rocchi, R.; Zappella, M.; Renieri, A. Real-time quantitative PCR as a routine method for screening large rearrangements in Rett syndrome: Report of one case of MECP2 deletion and one case of MECP2 duplication. Hum. Mutat. 2004, 24, 172-177. [CrossRef] [PubMed]

7. Van Esch, H. MECP2 Duplication Syndrome. In GeneReviews(R); Pagon, R.A., Adam, M.P., Ardinger, H.H., Wallace, S.E., Amemiya, A., Bean, L.J.H., Bird, T.D., Fong, C.T., Mefford, H.C., Smith, R.J.H., et al., Eds.; University of Washington: Seattle, WA, USA, 1993.

8. Nagarajan, R.P.; Hogart, A.R.; Gwye, Y.; Martin, M.R.; LaSalle, J.M. Reduced MeCP2 expression is frequent in autism frontal cortex and correlates with aberrant MECP2 promoter methylation. Epigenetics 2006, 1, 172-182. [CrossRef]

9. Nagarajan, R.P.; Patzel, K.A.; Martin, M.; Yasui, D.H.; Swanberg, S.E.; Hertz-Picciotto, I.; Hansen, R.L.; Van de Water, J.; Pessah, I.N.; Jiang, R.; et al. MECP2 promoter methylation and X chromosome inactivation in autism. Autism Res. 2008, 1, 169-178. [CrossRef] [PubMed]

10. Liyanage, V.R.; Jarmasz, J.S.; Murugeshan, N.; Del Bigio, M.R.; Rastegar, M.; Davie, J.R. DNA modifications: Function and applications in normal and disease States. Boilogy 2014, 3, 670-723. [CrossRef]

11. Liyanage, V.R.; Curtis, K.; Zachariah, R.M.; Chudley, A.E.; Rastegar, M. Overview of the Genetic Basis and Epigenetic Mechanisms that Contribute to FASD Pathobiology. Curr. Top. Med. Chem. 2017, 17, 808-828. [CrossRef] [PubMed]

12. Kim, K.C.; Choi, C.S.; Kim, J.W.; Han, S.H.; Cheong, J.H.; Ryu, J.H.; Shin, C.Y. MeCP2 Modulates Sex Differences in the Postsynaptic Development of the Valproate Animal Model of Autism. Mol. Neurobiol. 2016, 53, 40-56. [CrossRef]

13. Deng, X.; Berletch, J.B.; Nguyen, D.K.; Disteche, C.M. X chromosome regulation: Diverse patterns in development, tissues and disease. Nat. Rev. Genet. 2014, 15, 367-378. [CrossRef]

14. Lioy, D.T.; Garg, S.K.; Monaghan, C.E.; Raber, J.; Foust, K.D.; Kaspar, B.K.; Hirrlinger, P.G.; Kirchhoff, F.; Bissonnette, J.M.; Ballas, N. A role for glia in the progression of Rett's syndrome. Nature 2011, 475, 497-500. [CrossRef] [PubMed]

15. Zachariah, R.M.; Olson, C.O.; Ezeonwuka, C.; Rastegar, M. Novel MeCP2 isoform-specific antibody reveals the endogenous MeCP2E1 expression in murine brain, primary neurons and astrocytes. PLoS ONE 2012, 7, e49763. [CrossRef] [PubMed]

16. Ballas, N.; Lioy, D.T.; Grunseich, C.; Mandel, G. Non-cell autonomous influence of MeCP2-deficient glia on neuronal dendritic morphology. Nat. Neurosci. 2009, 12, 311-317. [CrossRef]

17. Liyanage, V.R.; Zachariah, R.M.; Rastegar, M. Decitabine alters the expression of Mecp2 isoforms via dynamic DNA methylation at the Mecp2 regulatory elements in neural stem cells. Mol. Autism 2013, 4, 46. [CrossRef]

18. Liyanage, V.R.; Zachariah, R.M.; Davie, J.R.; Rastegar, M. Ethanol deregulates Mecp2/MeCP2 in differentiating neural stem cells via interplay between 5-methylcytosine and 5-hydroxymethylcytosine at the Mecp2 regulatory elements. Exp. Neurol. 2015, 265, 102-117. [CrossRef]

19. Olson, C.O.; Zachariah, R.M.; Ezeonwuka, C.D.; Liyanage, V.R.; Rastegar, M. Brain region-specific expression of MeCP2 isoforms correlates with DNA methylation within Mecp2 regulatory elements. PLoS ONE 2014, 9, e90645. [CrossRef] [PubMed]

20. Xu, W.; Liyanage, V.R.B.; MacAulay, A.; Levy, R.D.; Curtis, K.; Olson, C.O.; Zachariah, R.M.; Amiri, S.; Buist, M.; Hicks, G.G.; et al. Genome-Wide Transcriptome Landscape of Embryonic Brain-Derived Neural Stem Cells Exposed to Alcohol with Strain-Specific Cross-Examination in BL6 and CD1 Mice. Sci. Rep. 2019, 9, 206. [CrossRef]

21. Loomes, R.; Hull, L.; Mandy, W.P.L. What Is the Male-to-Female Ratio in Autism Spectrum Disorder? A Systematic Review and Meta-Analysis. J. Am. Acad. Child Adolesc. Psychiatry 2017, 56, 466-474. [CrossRef] [PubMed]

22. Fombonne, E. Epidemiology of pervasive developmental disorders. Pediatr. Res. 2009, 65, 591-598. [CrossRef]

23. Rastegar, M.; Hotta, A.; Pasceri, P.; Makarem, M.; Cheung, A.Y.; Elliott, S.; Park, K.J.; Adachi, M.; Jones, F.S.; Clarke, I.D.; et al. MECP2 isoform-specific vectors with regulated expression for Rett syndrome gene therapy. PLoS ONE 2009, 4, e6810. [CrossRef] 
24. Barber, B.A.; Liyanage, V.R.; Zachariah, R.M.; Olson, C.O.; Bailey, M.A.; Rastegar, M. Dynamic expression of MEIS1 homeoprotein in E14.5 forebrain and differentiated forebrain-derived neural stem cells. Ann. Anat. 2013, 195, 431-440. [CrossRef]

25. Cerase, A.; Pintacuda, G.; Tattermusch, A.; Avner, P. Xist localization and function: New insights from multiple levels. Genome Boil. 2015, 16, 166. [CrossRef]

26. Dewing, P.; Chiang, C.W.; Sinchak, K.; Sim, H.; Fernagut, P.O.; Kelly, S.; Chesselet, M.F.; Micevych, P.E.; Albrecht, K.H.; Harley, V.R.; et al. Direct regulation of adult brain function by the male-specific factor SRY. Curr. Biol. 2006, 16, 415-420. [CrossRef]

27. Lambert, J.-F.; Benoit, B.O.; Colvin, G.A.; Carlson, J.; Delville, Y.; Quesenberry, P.J. Quick sex determination of mouse fetuses. J. Neurosci. Methods 2000, 95, 127-132. [CrossRef]

28. Patrat, C.; Okamoto, I.; Diabangouaya, P.; Vialon, V.; Le Baccon, P.; Chow, J.; Heard, E. Dynamic changes in paternal X-chromosome activity during imprinted X-chromosome inactivation in mice. Proc. Natl. Acad. Sci. USA 2009, 106, 5198-5203. [CrossRef]

29. Edgar, R.; Tan, P.P.C.; Portales-Casamar, E.; Pavlidis, P. Meta-analysis of human methylomes reveals stably methylated sequences surrounding $\mathrm{CpG}$ islands associated with high gene expression. Epigenet. Chromatin 2014, 7, 28. [CrossRef]

30. Doi, A.; Park, I.-H.; Wen, B.; Murakami, P.; Aryee, M.J.; Irizarry, R.; Herb, B.; Ladd-Acosta, C.; Rho, J.; Loewer, S. Differential methylation of tissue-and cancer-specific CpG island shores distinguishes human induced pluripotent stem cells, embryonic stem cells and fibroblasts. Nat. Genet. 2009, 41, 1350-1353. [CrossRef]

31. Irizarry, R.A.; Ladd-Acosta, C.; Wen, B.; Wu, Z.; Montano, C.; Onyango, P.; Cui, H.; Gabo, K.; Rongione, M.; Webster, M. The human colon cancer methylome shows similar hypo-and hypermethylation at conserved tissue-specific CpG island shores. Nat. Genet. 2009, 41, 178-186. [CrossRef] [PubMed]

32. Van Veldhoven, K.; Polidoro, S.; Baglietto, L.; Severi, G.; Sacerdote, C.; Panico, S.; Mattiello, A.; Palli, D.; Masala, G.; Krogh, V.; et al. Epigenome-wide association study reveals decreased average methylation levels years before breast cancer diagnosis. Clin. Epigenet. 2015, 7, 67. [CrossRef]

33. Hansen, K.D.; Timp, W.; Bravo, H.C.; Sabunciyan, S.; Langmead, B.; McDonald, O.G.; Wen, B.; Wu, H.; Liu, Y.; Diep, D. Increased methylation variation in epigenetic domains across cancer types. Nat. Genet. 2011, 43, 768-775. [CrossRef]

34. Huang, W.-Y.; Hsu, S.-D.; Huang, H.-Y.; Sun, Y.-M.; Chou, C.-H.; Weng, S.-L.; Huang, H.-D. MethHC: A database of DNA methylation and gene expression in human cancer. Nucleic Acids Res. 2014, 43, D856-D861. [CrossRef] [PubMed]

35. Huang, W.Y.; Hsu, S.D.; Huang, H.Y.; Sun, Y.M.; Chou, C.H.; Weng, S.L.; Huang, H.D. MethHC: A Database of DNA Methylation and Gene Expression in Human Cancer. Available online: http://methhc.mbc.nctu.edu. tw/php/index.php (accessed on 6 February 2017).

36. Vieira, J.P.; Lopes, F.; Silva-Fernandes, A.; Sousa, M.V.; Moura, S.; Sousa, S.; Costa, B.M.; Barbosa, M.; Ylstra, B.; Temudo, T.; et al. Variant Rett syndrome in a girl with a pericentric X-chromosome inversion leading to epigenetic changes and overexpression of the MECP2 gene. Int. J. Dev. Neurosci. 2015, 46, 82-87. [CrossRef] [PubMed]

37. Delcuve, G.P.; Rastegar, M.; Davie, J.R. Epigenetic control. J. Cell. Physiol. 2009, 219, 243-250. [CrossRef]

38. Kurian, J.R.; Forbes-Lorman, R.M.; Auger, A.P. Sex Difference in Mecp2 Expression During a Critical Period of Rat Brain Development. Epigenetics 2007, 2, 173-178. [CrossRef] [PubMed]

39. Berletch, J.B.; Ma, W.; Yang, F.; Shendure, J.; Noble, W.S.; Disteche, C.M.; Deng, X. Escape from X inactivation varies in mouse tissues. PLoS Genet. 2015, 11, e1005079. [CrossRef] [PubMed]

40. Khalil, A.M.; Driscoll, D.J. Trimethylation of histone H3 lysine 4 is an epigenetic mark at regions escaping mammalian X inactivation. Epigenetics 2007, 2, 114-118. [CrossRef] [PubMed]

41. Nino-Soto, M.; Nuber, U.A.; Basrur, P.; Ropers, H.-H.; King, W. Differences in the pattern of X-linked gene expression between fetal bovine muscle and fibroblast cultures derived from the same muscle biopsies. Cytogenet. Genome Res. 2005, 111, 57-64. [CrossRef] [PubMed]

42. Talebizadeh, Z.; Simon, S.D.; Butler, M.G. X chromosome gene expression in human tissues: Male and female comparisons. Genomics 2006, 88, 675-681. [CrossRef] 
43. Rastegar, M. Epigenetics and Cerebellar Neurodevelopmental Disorders. In Development of the Cerebellum from Molecular Aspects to Diseases; Marzban, H., Ed.; Springer International Publishing: Cham, Switzerland, 2017; pp. 197-218.

44. Marzban, H.; Del Bigio, M.R.; Alizadeh, J.; Ghavami, S.; Zachariah, R.M.; Rastegar, M. Cellular commitment in the developing cerebellum. Front. Cell. Neurosci. 2015, 8, 450. [CrossRef]

45. Shukla, S.; Kavak, E.; Gregory, M.; Imashimizu, M.; Shutinoski, B.; Kashlev, M.; Oberdoerffer, P.; Sandberg, R.; Oberdoerffer, S. CTCF-promoted RNA polymerase II pausing links DNA methylation to splicing. Nature 2011, 479, 74-79. [CrossRef] [PubMed]

46. Huang, Y.; Pastor, W.A.; Shen, Y.; Tahiliani, M.; Liu, D.R.; Rao, A. The behaviour of 5-hydroxymethylcytosine in bisulfite sequencing. PLoS ONE 2010, 5, e8888. [CrossRef]

47. Qui, Y.; Yang, Q.; Sui, F.; Lu, R.; Dang, S.; Ji, M.; He, N.; Shi, B.; Hou, P. A Strategy for Accurate Quantification of 5-Methylcytosine and 5-Hydroxymethylcytosine at CpG Sites Within Gene Promoter. J. Biomed. Nanotechnol. 2015, 11, 1016-1026. [CrossRef] [PubMed]

48. Guo, J.U.; Su, Y.; Shin, J.H.; Shin, J.; Li, H.; Xie, B.; Zhong, C.; Hu, S.; Le, T.; Fan, G. Distribution, recognition and regulation of non-CpG methylation in the adult mammalian brain. Nat. Neurosci. 2014, 17, $215-222$. [CrossRef]

49. Yu, D.; Sakurai, F.; Corey, D.R. Clonal Rett Syndrome cell lines to test compounds for activation of wild-type MeCP2 expression. Bioorg. Med. Chem. Lett. 2011, 21, 5202-5205. [CrossRef] [PubMed]

50. Zhou, P.; Lu, Y.; Sun, X.-H. Zebularine suppresses TGF-beta-induced lens epithelial cell-myofibroblast transdifferentiation by inhibiting MeCP2. Molecular 2011, 17, 2717.

51. He, S.; Barron, E.; Ishikawa, K.; Khanamiri, H.N.; Spee, C.; Zhou, P.; Kase, S.; Wang, Z.; Dustin, L.D.; Hinton, D.R. Inhibition of DNA Methylation and Methyl-CpG-Binding Protein 2 Suppresses RPE Transdifferentiation: Relevance to Proliferative VitreoretinopathyDNA Methylation Regulates RPE Transdifferentiation. Investig. Ophthalmol. Vis. Sci. 2015, 56, 5579-5589. [CrossRef]

52. Hartshorn, C.; Rice, J.E.; Wangh, L.J. Developmentally-regulated changes of Xist RNA levels in single preimplantation mouse embryos, as revealed by quantitative real-time PCR. Mol. Reprod. Dev. 2002, 61, 425-436. [CrossRef]

(C) 2019 by the authors. Licensee MDPI, Basel, Switzerland. This article is an open access article distributed under the terms and conditions of the Creative Commons Attribution (CC BY) license (http://creativecommons.org/licenses/by/4.0/). 